\title{
Development of a nomogram for screening of hepatitis B virus- associated hepatocellular carcinoma
}

\author{
Jung Wha Chung ${ }^{1, *}$, Eun Sun Jang ${ }^{1, *}$, Jaihwan Kim ${ }^{1}$, Sook-Hyang Jeong ${ }^{1,2}$, Nayoung \\ $\mathrm{Kim}^{1,2}$, Dong Ho Lee ${ }^{1,2}$, Kyung Ho Lee ${ }^{3}$ and Jin-Wook Kim ${ }^{1,2}$ \\ ${ }^{1}$ Department of Medicine, Seoul National University Bundang Hospital, Seongnam, Republic of Korea \\ ${ }^{2}$ Department of Internal Medicine, Seoul National University College of Medicine, Seoul, Republic of Korea \\ ${ }^{3}$ Department of Radiology, Seoul National University College of Medicine, Seoul, Republic of Korea \\ "These authors contributed equally to this work \\ Correspondence to: Jin-Wook Kim, email: kimjw@snubh.org \\ Keywords: chronic hepatitis B; hepatocellular carcinoma; nomograms; early diagnosis of cancer; ultrasonography \\ Received: August 06, $2017 \quad$ Accepted: October 28, $2017 \quad$ Published: November 18, 2017 \\ Copyright: Chung et al. This is an open-access article distributed under the terms of the Creative Commons Attribution License \\ 3.0 (CC BY 3.0), which permits unrestricted use, distribution, and reproduction in any medium, provided the original author and \\ source are credited.
}

\section{ABSTRACT}

Current strategy of hepatocellular carcinoma (HCC) surveillance evaluates individual risks of HCC for defining candidates for surveillance, but estimated risks are not utilized for clinical decision-making during actual screening. We sought to determine whether consideration of individual risks improve the performance of ultrasound (US)-based HCC screening in a real-world chronic hepatitis B (CHB) cohort. This single center retrospective cohort study analyzed 27,722 screening US tests from 4,175 consecutive CHB patients. Logistic regression analysis was performed to identify independent parameters predicting presence of HCC. A nomogram was built based on the independent predictors of HCC and compared with US-only screening by receiver operating characteristics analysis. The cost-effectiveness of the nomogram was assessed by decision curve analysis. HCC developed in 222 patients with the incidence of 0.769 per 1000 person-year during the median follow-up of 63 months. Age, sex, presence of cirrhosis, serum alpha-fetoprotein (AFP) levels and positive US test results were independent predictors of HCC presence. A nomogram based on these predictors showed higher C-statistics compared to US-only screening $\mathbf{0 . 9 6 0}$ vs. 0.731 and 0.935 vs. 0.691 for derivation and validation cohort, respectively; $p<0.001$ ). Decision curve analysis showed higher net benefit of the HCC nomogramguided screening model compared to US-only screening in the risk threshold range between 0 and 0.3. A nomogram composed of age, sex, presence of cirrhosis, serum AFP levels and US findings better predicted the presence of HCC compared to US-only screening in CHB on surveillance.

\section{INTRODUCTION}

Chronic hepatitis B virus infection is one of the leading causes of hepatocellular carcinoma (HCC) worldwide [1]. Surveillance for HCC is recommended for chronic hepatitis B (CHB) patients with increased risks [2-4], and ultrasonography (US) is a universally recommended screening test for HCC surveillance [3-5]. There have been concerns, however, about the sensitivity of screening US for small HCC in CHB, especially in the presence of regenerative nodules and fibrous septa [6-8]. Dynamic imaging techniques such as 4-phase multidetector computed tomography (CT) and dynamic contrast enhanced magnetic resonance imaging (MRI) have better sensitivity for small HCC compared to US [9], and the high specificity of dynamic imaging techniques obviate the need for biopsy when typical enhancing patterns are observed [10]. Considering the radiation hazards and high cost, however, dynamic imaging modalities are reserved for occasions when screening 
US suggests possibility of $\mathrm{HCC}[3,5]$ or technical issues hamper optimal US evaluation $[3,11]$.

Bayesian theorem indicates that the post-test probability of a disease is determined by pre-test disease probability and likelihood ratio of the corresponding test $[12,13]$. From the Bayesian perspective, the HCC probability of a CHB patient on surveillance is dependent not only on the results of the screening US but also on the baseline probability of HCC. Clinical and laboratory parameters such as age, sex, ethnicity, hepatitis B virus (HBV) viral loads, presence of cirrhosis and elevated alphafetoprotein (AFP) levels have been validated for predicting the risk for HCC incidence [14-19], and it is suggested that these parameters may also estimate the probability for immediate development of HCC [20]. Current guidelines employ these risk predictors in defining at-risk population for surveillance, but individual risks are not considered in the decision to implement an enhanced follow-up or to trigger a recall policy during surveillance [3-5].

We speculated that predictors of long-term HCC risk may also be used for estimating the probability of $\mathrm{HCC}$ presence, and that integrating these predictors may improve the accuracy of the US-based screening. To test this hypothesis, we developed a nomogram predicting presence of $\mathrm{HCC}$ in a real-world $\mathrm{CHB}$ cohort on surveillance, and compared the screening performance of the nomogram with that of traditional US-only screening.

\section{RESULTS}

\section{Characteristics of study cohort}

The final cohort included 4,175 CHB patients, who were randomly allocated to the derivation set $(n=2,087)$ and the validation set $(n=2,088)$ (Figure 1). The characteristics of the two groups were similar at baseline and end of follow-up (Table 1). HCC developed in 222 patients with the incidence 0.769 per 1000 person-year during the median follow-up of 63 months $(95 \% \mathrm{CI}$ : 0.674-0.877). The HCC incidence was similar between the derivation and validation sets (Supplementary Figure 1A). Patients with cirrhosis had significantly higher HCC incidence compared to non-cirrhotic patients (2.94 vs. 0.17 per 1000 person-year, respectively, $p<0.001$; Supplementary Figure 1B). The stage of HCC was BCLC 0, A, B and C for 41, 43, 5.3 and $10.7 \%$ of cases, respectively. The median size of the largest nodule was $2.0 \mathrm{~cm}$ (IQR, $1.6 \mathrm{~cm}$ ). The main reason for advanced stage was involvement of portal vein: $83 \%$ of BCLC-C patients showed portal vein invasion.

\section{Age, sex, cirrhosis and serum AFP as independent predictors for presence of $\mathrm{HCC}$}

During the study period, 27,855 screening US tests were performed. After excluding 133 tests with 'ambiguous' association with $\mathrm{HCC}$ as described in the Methods, the remaining 13,908 and 13,814 tests from derivation and validation set were analyzed respectively. Logistic regression analysis of the 13,908 screening events in the derivation dataset identified factors predicting presence of HCC: new nodule(s) by US, old age, male sex, presence of cirrhosis, high AFP levels, high HBsAg titers, low albumin levels, high bilirubin levels, high AST levels, low platelet counts and prolonged prothrombin time were significant predictors of $\mathrm{HCC}$ presence (Table 2). Multivariate analysis selected old age, male sex, presence of cirrhosis and high AFP as independent predictors of HCC presence in addition to the positive US findings. Reclassification analysis also showed significant improvements in prediction of HCC presence by adding the four independent predictors to US-only screening, regardless of the stages of HCC (Table 3): the NRI of 1.31 and 1.29 for derivation and validation set, respectively, indicated about $65 \%(1 / 2(N R I)$ improvement in correct reclassification by the nomogram [21]. The positive IDIs also represented improved integrated difference in the corresponding Youden's indices by the nomogram [22].

\section{Development of a nomogram for predicting presence of $\mathrm{HCC}$}

Since the logistic analysis and reclassification analysis showed that traditional risk factors for HCC (age, sex, cirrhosis and AFP) provided additional information on the prediction of HCC presence, a nomogram was developed using the independent predictors to generate a combined indicator for estimating the probability of HCC presence (Figure 2). Calibration analysis showed that the HCC nomogram had good correlation between the predicted and observed probabilities within the clinically useful range $(0-0.3)$, beyond which the model overestimated the probability in the validation set (Figure 3). The overall goodness-of-fit test showed that the nomogram satisfactorily fitted the observed probabilities without significant deviation $(p=0.72$ and 0.82 for derivation and validation set, respectively, by HosmerLemeshow test).

\section{Performance of HCC nomogram: comparison of screening accuracy with US-only screening}

We then sought to determine whether the nomogram outperformed US-only screening strategy in predicting presence of HCC. The sensitivity and specificity of US was $47.1 \%$ and $99.1 \%$ in the derivation cohort, respectively, and $39.1 \%$ and $99.3 \%$ in the validation cohort, respectively: among the 222 patients who developed HCC, 73 cases were confirmed by CT or MR although screening US showed no evidence of new nodule(s). Compared to US-only screening, the HCC nomogram showed higher sensitivity with minimal 
trade-off of specificity (Table 3 ): at the cut-off of 140 , the HCC nomogram score had sensitivity and specificity of $62.9 \%$ and $98.7 \%$ in the derivation set, respectively, and $54.2 \%$ and $98.7 \%$ in the validation set, respectively. In cases with negative US tests, the nomogram showed $58-65 \%$ sensitivity at the specificity of $95 \%$ with the cut-off of 110-112 (Supplementary Table 1). ROC analysis demonstrated that the $C$-statistic value for the nomogram was significantly higher compared to US: 0.960 vs. $0.731(p<0.001)$, respectively, in the derivation dataset, and 0.935 vs. 0.691, respectively, in the validation dataset $(p<0.001)$ (Table 4). The superiority of HCC nomogram was significant regardless of tumor stages and history of nucleos(t)ide analog therapy.

\section{Cost-effectiveness of HCC nomogram-guided} screening model: decision curve analysis

Because the increased sensitivity of HCC nomogram was associated with small decrease in specificity, decision curve analysis was performed to determine whether the benefit of the nomogram was clinically useful in terms of cost-effectiveness. HCC nomogram-guided screening model had greater net benefit compared to the US-only screening in the risk threshold range between 0 and 0.3 , both in the derivation and validation dataset (Figure 4). This result suggested that HCC nomogram-guided decision to perform confirmatory tests may be clinically cost-effective for patients whose cost-benefit ratio, i.e., harms of confirmatory tests to harms of missing HCC, is less than $2 / 3$.

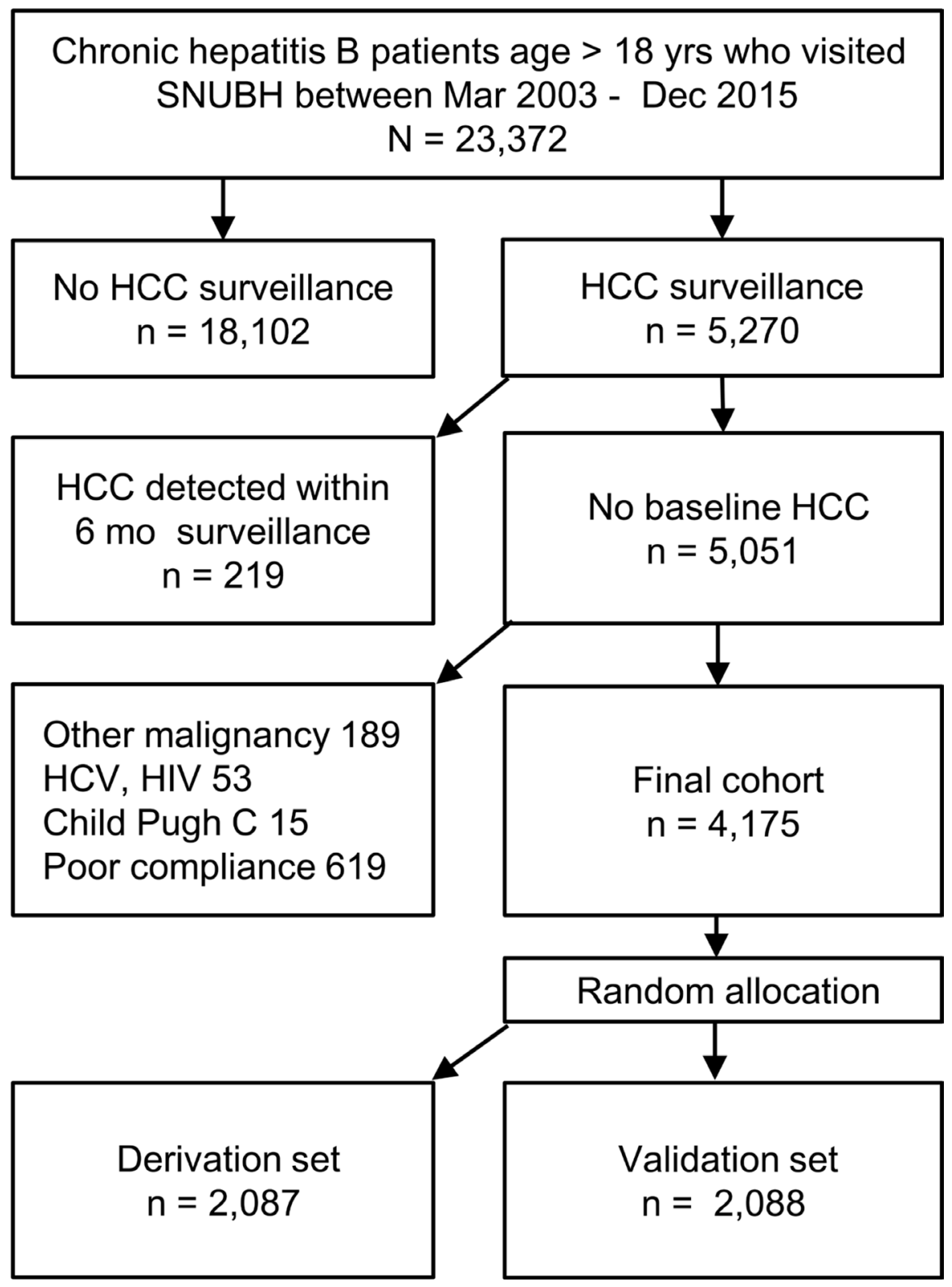

Figure 1: Participant flow diagram. 
Table 1: Characteristics of patients

\begin{tabular}{|c|c|c|c|c|c|c|}
\hline \multirow{2}{*}{ Parameter } & \multicolumn{3}{|c|}{ Baseline } & \multicolumn{3}{|c|}{ End of follow-up } \\
\hline & Derivation set & Validation set & $P$ value & Derivation set & Validation set & $P$ value \\
\hline Number of patients & 2,087 & 2,088 & - & 2,087 & 2,088 & - \\
\hline Follow-up, months & - & - & - & $62(76)$ & $63(74)$ & 0.60 \\
\hline Nucleos(t)ide analog (\%) & $644(31)$ & $631(30)$ & 0.66 & $1,059(51)$ & $1,053(50)$ & 0.84 \\
\hline Age, years & $45(16)$ & $45(13)$ & 0.13 & $52(17)$ & $52(16)$ & 0.12 \\
\hline Male (\%) & $1,202(58)$ & $1,285(60)$ & 0.12 & & & \\
\hline Liver cirrhosis $(\%)$ & $431(21)$ & $446(21)$ & 0.59 & & & \\
\hline HCC development (\%) & & & & $113(5.4)$ & $109(5.2)$ & 0.79 \\
\hline HBeAg positivity (\%) & $719(34)$ & $732(35)$ & 0.68 & 400 (19) & $429(21)$ & 0.12 \\
\hline HBs Ag (IU/mL) & $3831(4086)$ & $3832(3878)$ & 0.92 & 3577 (3386) & $3529(3135)$ & 0.68 \\
\hline HBV DNA (log IU/mL) & $3.7(3.9)$ & $3.6(3.8)$ & 0.34 & $1.8(1.5)$ & $1.8(1.6)$ & 0.10 \\
\hline Albumin (g/dL) & $4.3(0.4)$ & $4.3(0.4)$ & 0.69 & $4.4(0.4)$ & $4.5(0.3)$ & 0.95 \\
\hline Bilirubin (mg/dL) & $0.9(0.5)$ & $0.9(0.5)$ & 0.60 & $0.8(0.5)$ & $0.8(0.4)$ & 0.52 \\
\hline AST (IU/L) & $30(24)$ & $30(23)$ & 0.96 & $25(10)$ & $25(11)$ & 0.26 \\
\hline ALT (IU/L) & $35(39)$ & $35(38)$ & 0.29 & $24(17)$ & $24(16)$ & 0.05 \\
\hline Platelet $\left(\times 10^{9} / \mathrm{L}\right)$ & $191(79)$ & $192(75)$ & 0.76 & $200(78)$ & $201(77)$ & 0.65 \\
\hline Prothrombin time (INR) & $1.0(0.1)$ & $1.0(0.1)$ & 0.95 & $1.0(0.1)$ & $1.0(0.1)$ & 0.96 \\
\hline
\end{tabular}

Data are presented as median (interquartile range) or numbers (percent)

ALT, alanine aminotransferase; AST, aspartate aminotransferase; HCC, hepatocellular carcinoma; INR, international normalization ratio.

Table 2: Logistic regression analysis of predictors for presence of $\mathrm{HCC}$

\begin{tabular}{|c|c|c|c|c|}
\hline \multirow{2}{*}{$\begin{array}{l}n=13,908 \\
\text { Parameter }\end{array}$} & \multicolumn{2}{|c|}{ Univariate } & \multicolumn{2}{|c|}{ Multivariate } \\
\hline & OR $(95 \% \mathrm{CI})$ & $P$ value & OR $(95 \% \mathrm{CI})$ & $P$ value \\
\hline Positive US finding ${ }^{\mathrm{a}}$ & $101.1(61.1-167.1)$ & $<0.001$ & $38.5(10.4-142.1)$ & $<0.001$ \\
\hline Age (years) & $1.1(1.0-1.1)$ & $<0.001$ & $1.1(1.0-1.1)$ & 0.003 \\
\hline Male sex & $2.0(1.2-3.5)$ & $<0.001$ & $5.2(1.2-22.4)$ & 0.03 \\
\hline Liver cirrhosis & $13.4(7.4-24.7)$ & $<0.001$ & $7.2(1.5-33.8)$ & 0.012 \\
\hline AFP (Log ng/mL) & $7.6(5.8-10.1)$ & $<0.001$ & $19.4(7.8-48.6)$ & $<0.001$ \\
\hline HBeAg positivity & $1.0(0.6-1.7)$ & 0.90 & & \\
\hline HBsAg titer $(\log I U / m L)$ & $1.9(1.0-3.5)$ & 0.048 & $3.0(0.9-9.7)$ & 0.07 \\
\hline HBV DNA $(\log I U / m L)$ & $1.0(0.9-1.2)$ & 0.81 & & \\
\hline Nucleos(t)ide analog ${ }^{b}$ & $1.7(1.0-2.9)$ & 0.04 & $0.5(0.1-1.8)$ & 0.26 \\
\hline Albumin (g/dL) & $0.2(0.1-0.3)$ & $<0.001$ & $1.7(0.4-6.6)$ & 0.45 \\
\hline Bilirubin (mg/dL) & $1.2(1.1-1.4)$ & 0.006 & $0.6(0.3-1.5)$ & 0.30 \\
\hline $\mathrm{AST}>40 \mathrm{IU} / \mathrm{L}$ & $4.4(2.8-7.1)$ & $<0.001$ & $1.0(1.0-1.0)$ & 0.36 \\
\hline $\mathrm{ALT}>40 \mathrm{IU} / \mathrm{L}$ & $1.6(1.0-2.6)$ & 0.06 & & \\
\hline Platelet $\left(10^{9} / \mathrm{L}\right)$ & $1.0(0.9-1.0)$ & $<0.001$ & $1.0(0.02-1.0)$ & 0.07 \\
\hline Prothrombin time (INR) & $4.0(1.7-9.2)$ & 0.001 & $0.7(0.004-23.0)$ & 0.85 \\
\hline
\end{tabular}

Numbers in parenthesis indicate $95 \%$ CI obtained from 1000 bootstrapping iterations.

${ }^{a}$ Detection of nodule $(\mathrm{s})>1 \mathrm{~cm}$ which had not been previously characterized or showed changes in size or echo pattern.

${ }^{b}$ Exposure to nucleos(t)ide analog during study period.

AFP, alpha-fetoprotein; AST, aspartate aminotransaminase; ALT, alanine aminotransferase; INR. international normalized ratio; OR, odds ratio. 
Table 3: Reclassification, sensitivity and specificity of HCC screening models

\begin{tabular}{|c|c|c|c|c|}
\hline & \multicolumn{2}{|c|}{ Derivation set $(N=13,908)$} & \multicolumn{2}{|c|}{ Validation set $(N=13,814)$} \\
\hline & US-only & HCC nomogram & US-only & HCC nomogram \\
\hline \multicolumn{5}{|l|}{ All HCC } \\
\hline $\mathrm{NRI}^{a}$ & - & $1.31(1.17-1.52)$ & - & $1.29(1.05-1.49)$ \\
\hline $\operatorname{IDI}^{a}$ & - & $0.14(0.09-0.21)$ & - & $0.13(0.07-0.19)$ \\
\hline Sensitivity ${ }^{b}$ & $47.1(35.1-59.4)$ & $78.6(67.1-87.5)$ & $39.0(26.5-52.6)$ & $67.8(54.4-79.4)$ \\
\hline Specificity $^{b}$ & $99.1(99.0-99.3)$ & $96.1(95.7-96.4)$ & $99.2(99.1-99.4)$ & $95.9(95.6-96.2)$ \\
\hline Youden index & $0.463(0.349-0.577)$ & $0.784(0.703-0.829)$ & $0.382(0.263-0.501)$ & $0.745(0.637-0.815)$ \\
\hline \multicolumn{5}{|c|}{ BCLC 0/A HCC } \\
\hline $\mathrm{NRI}^{a}$ & - & $1.29(1.05-1.49)$ & - & $1.14(0.90-1.39)$ \\
\hline $\operatorname{IDI}^{a}$ & - & $0.13(0.07-0.19)$ & - & $0.07(0.02-0.13)$ \\
\hline Sensitivity $^{b}$ & $47.1(35.1-59.4)$ & $62.9(50.5-75.4)$ & $39.1(25.1-54.6)$ & $54.2(40.8-67.3)$ \\
\hline Specificity $^{b}$ & $99.1(99.0-99.3)$ & $98.7(98.5-98.9)$ & $99.3(99.1-99.4)$ & $98.7(98.5-98.9)$ \\
\hline Youden index & $0.463(0.349-0.577)$ & $0.777(0.707-0.812)$ & $0.384(0.263-0.501)$ & $0.741(0.624-0.812)$ \\
\hline
\end{tabular}

$N$ indicates the numbers of HCC screening tests performed during the study period; numbers in parenthesis indicate $95 \%$ confidence intervals.

amprovements by adding age, sex, cirrhosis and AFP levels to US by continuous net reclassification improvement (NRI) and integrated discrimination improvement (IDI) analyses with 300 bootstrapping iterations.

${ }^{\mathrm{b}}$ Confidence interval from 1000 bootstrapping iterations; cut-off value of 140 for HCC nomogram score.

\section{DISCUSSION}

In this study, we demonstrated that consideration of individual risks helps predicting presence of $\mathrm{HCC}$ in a real-world $\mathrm{CHB}$ cohort on surveillance: the HCC nomogram which integrated the HCC risk predictors with the results of screening US had superior accuracy compared to US-only screening. The nomogram-guided screening model also showed cost-effectiveness by decision curve analysis.
The reported sensitivity of US for early HCC vary among studies [23], but recent studies suggested relatively low sensitivity in the surveillance setting [7, 8, 24]. Our data also revealed similarly low sensitivity of US in CHB. Advances in the dynamic imaging technology allows imaging diagnosis of smaller HCCs [2, 5], and it can be speculated that the sensitivity of US may decrease as HCC is diagnosed in the earlier stage [9]. In addition, we classified serial screening data up to 6 months before final diagnosis of

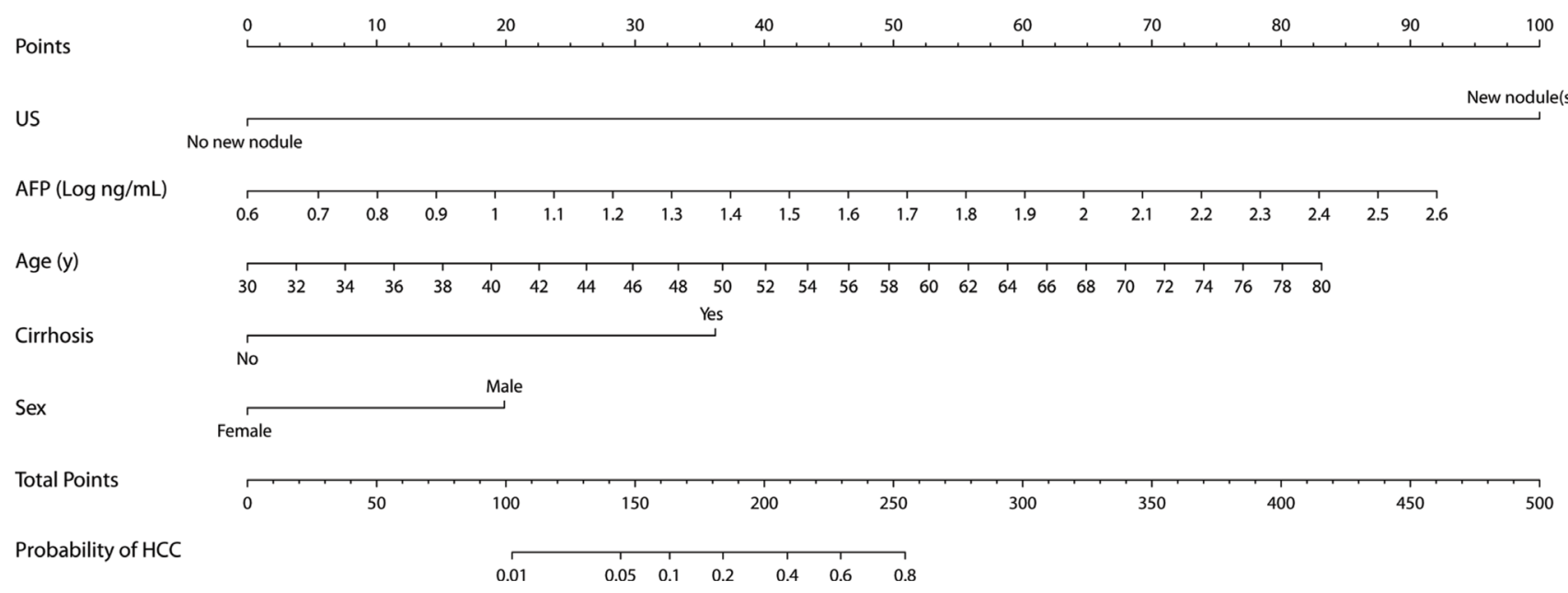

Figure 2: Nomogram for predicting presence of HCC in chronic hepatitis B patients on surveillance. The individual point score for each variable is obtained on the corresponding perpendicular position on the top "Points" axis. Continuous values, i.e. age and $\log$ AFP, outside of the boundaries are replaced by the corresponding boundary value. The sum of all points, HCC nomogram scores, are converted to predicted HCC probability on the bottom probability axis. 

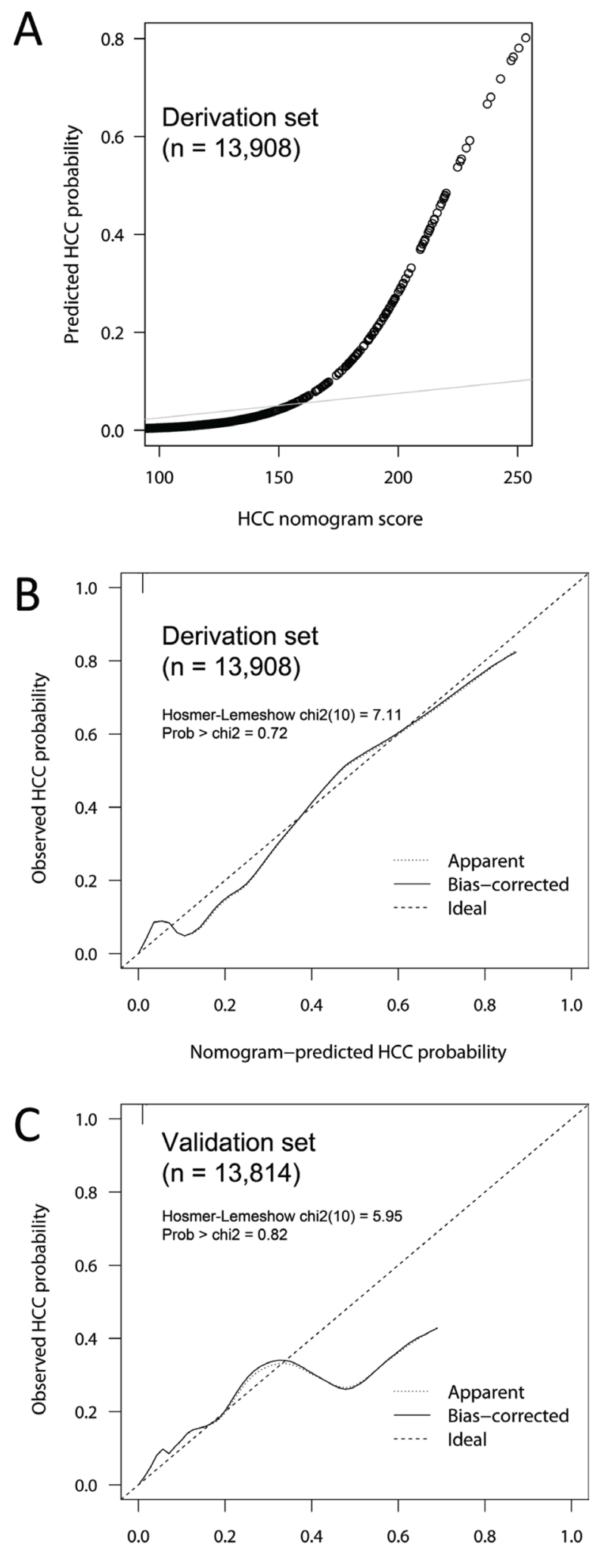

Nomogram-predicted HCC probability

Figure 3: Calibration of the HCC nomogram score model. The predicted probability of HCC presence was plotted against HCC nomogram score in the derivation dataset (A) Agreement between the predicted and observed HCC probabilities were plotted for the derivation (B) and validation (C) datasets with 300 bootstraps. The HCC nomogram score showed good calibration within the expected probability range up to 0.3 , which corresponds to HCC nomogram score of 195. Hosmer and Lemeshow's goodness-of-fit test showed no significant discrepancies between the predicted and observed probabilities for HCC presence $(p=0.72$ and 0.82 for derivation and validation set, respectively). 
Table 4: Comparison of areas under receiver operating characteristic curves between US- and HCC nomogram-based HCC screening

\begin{tabular}{|c|c|c|c|c|c|c|c|c|}
\hline \multirow[b]{2}{*}{ All HCC } & \multicolumn{4}{|c|}{ Derivation set } & \multicolumn{4}{|c|}{ Validation set } \\
\hline & $N$ & US-only & HCC nomogram & $P$ value & $N$ & US-only & HCC nomogram & $P$ value \\
\hline Total & 13,908 & $\begin{array}{c}0.731 \\
(0.724-0.739)\end{array}$ & $\begin{array}{c}0.960 \\
(0.956-0.963)\end{array}$ & $<0.001$ & 13,814 & $\begin{array}{c}0.691 \\
(0.683-0.699)\end{array}$ & $\begin{array}{c}0.935 \\
(0.931-0.939)\end{array}$ & $<0.001$ \\
\hline $\mathrm{NA}(+)$ & 8,208 & $\begin{array}{c}0.725 \\
(0.715-0.735)\end{array}$ & $\begin{array}{c}0.943 \\
(0.938-0.948)\end{array}$ & $<0.001$ & 7,997 & $\begin{array}{c}0.698 \\
(0.687-0.708)\end{array}$ & $\begin{array}{c}0.913 \\
(0.907-0.919)\end{array}$ & $<0.001$ \\
\hline $\mathrm{NA}(-)$ & 5,700 & $\begin{array}{c}0.746 \\
(0.735-0.758)\end{array}$ & $\begin{array}{c}0.985 \\
(0.981-0.988)\end{array}$ & $<0.001$ & 5,817 & $\begin{array}{c}0.674 \\
(0.662-0.686)\end{array}$ & $\begin{array}{c}0.967 \\
(0.963-0.972)\end{array}$ & $<0.001$ \\
\hline BCLC 0/A & $N$ & US-only & HCC nomogram & $P$ value & $N$ & US-only & HCC nomogram & $P$ value \\
\hline Total & 13,833 & $\begin{array}{c}0.741 \\
(0.734-0.749)\end{array}$ & $\begin{array}{c}0.956 \\
(0.952-0.959)\end{array}$ & $<0.001$ & 13,717 & $\begin{array}{c}0.692 \\
(0.684-0.700)\end{array}$ & $\begin{array}{c}0.943 \\
(0.939-0.947)\end{array}$ & $<0.001$ \\
\hline $\mathrm{NA}(+)$ & 8,147 & $\begin{array}{c}0.721 \\
(0.711-0.731)\end{array}$ & $\begin{array}{c}0.938 \\
(0.933-0.943)\end{array}$ & $<0.001$ & 7,907 & $\begin{array}{c}0.702 \\
(0.692-0.712)\end{array}$ & $\begin{array}{c}0.925 \\
(0.919-0.931)\end{array}$ & $<0.001$ \\
\hline $\mathrm{NA}(-)$ & 5,686 & $\begin{array}{c}0.791 \\
(0.780-0.801)\end{array}$ & $\begin{array}{c}0.983 \\
(0.979-0.986)\end{array}$ & $<0.001$ & 5,810 & $\begin{array}{c}0.674 \\
(0.662-0.686)\end{array}$ & $\begin{array}{c}0.968 \\
(0.963-0.972)\end{array}$ & $<0.001$ \\
\hline
\end{tabular}

$N$ indicates the number of $\mathrm{HCC}$ screening tests performed during the study period.

The numbers in parenthesis indicate $95 \%$ confidence intervals.

NA (+) and NA (-) denote patients with or without exposure to nucleos(t)ide analog therapy during the study period.

All HCC: total patients; BCLC 0/A: patients without development of advanced HCC, i.e. BCLC B/C/D.

HCC as HCC-associated US, and this classification scheme may also have contributed to the low sensitivity.

Several studies have shown that age, sex, cirrhosis and AFP levels are predictors for long-term HCC risk in CHB [14-20, 25, 26]. A recent Korean multicenter cohort study also confirmed the long-term predictive significance

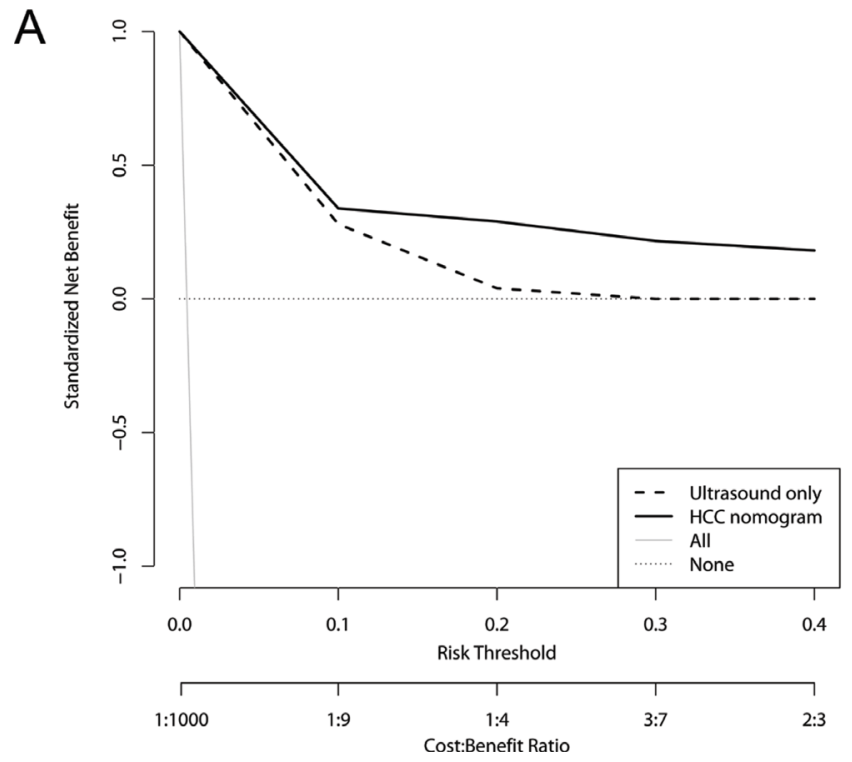

of age, sex and cirrhosis in CHB patients on oral NA therapy [26]. Our data demonstrated that these predictors can be used to estimate the probability of presence of HCC as well. This finding is in line with the recent report in which age, AFP, platelets, and alanine aminotransferase (ALT) predicted immediate development of HCC [20].

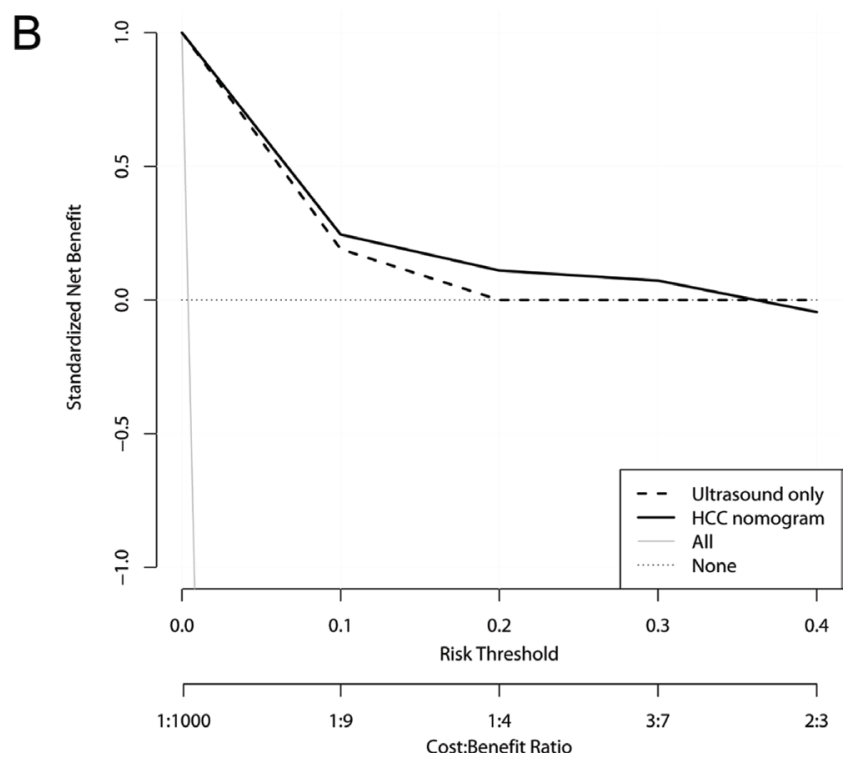

Figure 4: Decision curve analysis for HCC screening models. Decision curves for derivation set (A) and validation set (B). Ultrasound-only indicates traditional US-based screening in which decisions to request confirmatory tests are guided only by positive US tests. HCC nomogram indicates that the decisions are guided by HCC nomogram scores. Risk threshold and cost:benefit ratio indicate the relative significance of correct detection of HCC to correct exclusion of HCC of the models. All indicates that all CHB patients receive confirmatory tests, i.e. dynamic imaging studies or biopsy, and None indicates that no patients receive confirmatory tests. The net benefit of HCC nomogram was higher than that of US-only across given range of threshold probabilities, indicating that nomogram-based screening model would produce cost-effective clinical outcome irrespective of patient preference. 
The unique strength of our study was the inclusion of US test results in the multivariate analysis, which suggested that the four predictors may supplement US-based HCC screening. Since US is the only recommended screening tool for HCC, confirmatory dynamic imaging studies are triggered only by positive US findings and underlying risks of HCC are not reflected in the clinical decisions once surveillance is started under current recommendations $[2,3]$. Our HCC nomogram showed good calibration profile in predicting presence of $\mathrm{HCC}$ within the clinically relevant range, beyond which the confirmatory tests would be warranted without considering the nomogram scores. Furthermore, the HCC nomogram had significantly superior reclassification and discrimination characteristics over US-only screening, regardless of HCC stages. Taken together, it can be suggested that consideration of age, sex, status of cirrhosis and AFP levels along with US results improves the screening accuracy of HCC detection, probably by identifying additional patients for whom dynamic imaging studies are likely to produce positive results [27]. Long-term prospective validation is needed, however, to determine whether personalized surveillance based on HCC nomogram improves the performance and outcome of HCC surveillance in CHB.

Current AASLD and EASL guidelines do not recommend AFP as a screening test, mainly because of low sensitivity and specificity [2, 3, 28, 29]. Hepatitis activity may elevate AFP levels in CHB [30, 31], and elevated AFP levels may just signify increased risk for future development of HCC [18, 20, 29, 32]. However, AFP comprised one of the major components of our nomogram. Hepatitis activity may decrease over the course of CHB, either with or without NA therapy, and false positivity of AFP may decrease accordingly [33]. In our cohort, the median ALT level was $24 \mathrm{IU} / \mathrm{L}$ with IQR of 17 at the end of follow-up, and HBV DNA levels also showed decreasing tendency. Patients with hepatitis flare were likely to start NA therapy during the study period, as suggested in Table 1. Because we analyzed all of the serial screening data rather than baseline or final ones, AFP values associated with hepatitis flare may not have significantly affected the performance of the nomogram. This explanation is also in line with the finding that the superiority of nomogram over US was independent of exposure to NA therapy. The fact that elevated AFP may imply both high baseline risk and presence of HCC may not necessarily disprove its role, but rather render AFP suitable for our probability-oriented nomogram.

Currently AFP is seldom used alone in HCC surveillance, and there have been several reports indicating increase in the sensitivity of US-based surveillance by adding AFP [7, 34]. However, previous cost analyses provide no solid evidence supporting the combined use of AFP, and the benefit may be offset by increased false positivity and the following costs of confirmatory tests [7, 11, 34-36]. To address the same issue in our data, we performed the decision curve analysis, which revealed that the HCC nomogrambased screening model had greater net benefit compared to US-only model in the clinically appropriate risk threshold range [37], indicating that HCC nomogram-guided screening model may be cost-effective and can be recommended for all CHB patients with reasonable threshold probabilities without compromising specificity [38].

The main limitation of our study is retrospective design in single center. Consecutive patient was recruited from the comprehensive electronic registry [39-41] to reduce selection bias, and we adopted a split-sample approach with bootstrapping to reduce the possibility of overfitting. Nevertheless, our findings need further external validation in prospective settings. Secondly, our simulated decision curve analysis needs to be validated by including the performances of confirmatory imaging tests and cost-effectiveness parameters which are specific to regional settings. Lastly, the final outcome measurements of prospective validation studies need to include improvement of survival.

In conclusion, a nomogram composed of age, sex, presence of cirrhosis, serum AFP levels and US findings better predicts the probability of presence of $\mathrm{HCC}$ compared to US-only screening in CHB on surveillance. $\mathrm{HCC}$ nomogram-based screening has superior performance compared to US-only screening and cost-effective. The clinical usefulness of HCC nomogram-guided surveillance strategy needs to be validated in prospective studies.

\section{MATERIALS AND METHODS}

\section{Study population}

This single center retrospective cohort study enrolled consecutive $\mathrm{CHB}$ patients who were over age eighteen and underwent regular surveillance for $\mathrm{HCC}$ between Mar. 2003 and Dec. 2015 in a tertiary referral center in South Korea. Clinical and laboratory data were retrieved from the liver disease registry of Seoul National University Bundang Hospital Clinical Data Warehouse $[40,42]$. Patients with detection of HCC within 6 months after initial screening, malignancy other than $\mathrm{HCC}$, hepatitis $\mathrm{C}$ virus or human immunodeficiency virus coinfection, Child-Pugh class $\mathrm{C}$ or non-compliance of surveillance were excluded (Figure 1).

Screening US examination was carried out every 6-12 months along with blood tests including serum AFP levels, transaminases, prothrombin time, albumin, bilirubin and platelet counts. The presence of liver cirrhosis was defined by ultrasonographic features (coarse liver echotexture with nodularity) plus evidence of portal hypertension including ascites, splenomegaly, thrombocytopenia $\left(<100 \times 10^{9} / \mathrm{L}\right)$ and varices $[15,43]$. US findings were classified dichotomously as "positive" if nodule(s) $>1 \mathrm{~cm}$ was detected which had not been previously characterized or showed changes in size or echo 
pattern [2], or "no evidence of HCC" otherwise. When US detected suspicious nodule(s), multidetector 4-phase CT or MRI was performed. Dynamic imaging studies were also considered at the attending physicians' discretion in the case of successive rise in serum AFP levels, incomplete US examination due to poor sonic window, or very coarse echo-pattern with numerous nodules [3, 11]. Diagnosis of HCC was made according to the AASLD criteria [2].

The institutional review board of our hospital approved this study (IRB No: B-1609/361102). All clinical investigation has been conducted according to the principles expressed in the Declaration of Helsinki. Informed consent was wavered by IRB, due to the retrospective observational nature of study and anonymous analysis of data.

\section{Data collection and imputation}

In patients who developed $\mathrm{HCC}$, the US examinations up to 6 months before the confirmatory test were classified as "associated with HCC" [20], whereas US examinations performed more than 12 months before the confirmatory test were classified as "not associated with HCC" and analyzed as such. US examinations 6-12 months before confirmatory test were classified as "ambiguous" and excluded from the logistic regression analysis. Blood tests performed within 45 days before or 15 days after each US examination were linked to the corresponding US results and data outside of this range were excluded. Missing AFP data were predicted by multiple imputations with the number of imputations of 10 using bootstrap and predictive mean matching by $\mathrm{R}$ aregImpute package. The variables included in the multiple imputation model were previously identified as significant predictors of AFP elevation: presence of cirrhosis, AST, ALT, albumin, prothrombin time, HBV DNA levels and history of nucleos(t)ide analogues [41]. HCC was excluded from the imputation model, however, for the conservative estimation of the predictive role of AFP.

\section{Development and validation of a model predicting presence of $\mathrm{HCC}$}

Logistic regression analysis was used to identify predictors for presence of HCC at the time of each US measurement. For the internal validation, the final patients were randomly allocated to either derivation or validation dataset (Figure 1), and bootstrapping procedure was performed [44]. Predictor variables were selected by backward stepwise selection with a $P$ value $>0.05$ for removal. The outcome variable of the logistic model was presence of HCC. Reclassification analysis was performed by continuous net reclassification improvement (NRI) and integrated discrimination improvement (IDI) index to determine whether addition of independent predictors improve the prediction of present HCC $[21,22]$.
A nomogram was established from the independent predictors of the logistic model. The endpoint of the nomogram was detection of $\mathrm{HCC}$ at the time of US examination. The HCC nomogram score, the point sum of each parameter, was tested for calibration and discrimination [22, 45]. Calibration of the nomogram was evaluated for correct detection of HCC by calibration curves and Hosmer-Lemeshow goodness-of-fit test [46]. The discriminative ability was assessed by the receiver operating characteristic (ROC) curve analysis with comparison of concordance $(C)$ statistic [47].

\section{Decision curve analysis}

In order to determine whether HCC nomogram is cost-effective compared to US-only screening, we employed decision curve analysis. Decision curve analysis takes misclassification costs of diagnostic tests into account without assuming pre-defined utility, and balances the benefit of true positivity against the cost of false positivity of diagnostic tests $[37,38]$. The threshold probability is defined as the probability where the expected benefit of opting for confirmatory tests is equal to the expected benefit of avoiding confirmatory tests [38, 47]. For example, the risk threshold of 0.1 corresponds to cost-benefit ratio of $1: 9[0.1 /(1-0.1)]$ and signifies that a rational patient with this risk threshold will opt for $\mathrm{HCC}$ nomogram-based screening instead of US-only screening if the expected probability of having HCC is 0.1 or greater because the harms associated with a missed HCC is nine times greater than the harms associated with unnecessary additional tests. Net benefit indicates the difference between proportions of true positive and false positive, weighted by the risk threshold [38].

\section{Statistical analysis}

Statistical analyses performed using STATA version 14 (College Station, Texas) and $\mathrm{R}$ package (version 3.3.2, www.r-project.org). Continuous and categorical variables were tested by Student's $t$-test and $\chi^{2}$ test, respectively. Kaplan-Meier analysis was used to calculate the cumulative incidence of HCC of the study population. Generation of nomogram and calibration analysis was performed by $\mathrm{R} r m s$ package. Comparison of ROC curves was made by STATA ROCgold command. Reclassification analysis was performed by STATA incrisk command. Decision curve analysis was performed by $\mathrm{R}$ DecisionCurve package.

\section{Abbreviations}

HCC, hepatocellular carcinoma; CHB, chronic hepatitis B; US, ultrasonography; CT, computed tomography; MRI, magnetic resonance imaging; HBV, hepatitis B virus; AFP, alpha-fetoprotein; NRI, net 
reclassification improvement; IDI, integrated discrimination improvement; ROC, receiver operating characteristic; ALT, alanine aminotransferase.

\section{Author contributions}

Study concept and design: JWK ESJ JWC KHL; acquisition of data: JWK ESJ JWC; analysis and interpretation of data: JWK ESJ JWC JHH SHJ NK DHL KHL; drafting of the manuscript: JWK ESJ JWC HY JK CMS YSP; critical revision of the manuscript for important intellectual content: ESJ JWC KHL; statistical analysis: JWK ESJ JWC; obtained funding: JWK; study supervision: JWK.

\section{ACKNOWLEDGMENTS}

None.

\section{CONFLICTS OF INTEREST}

The authors have nothing to declare.

\section{FUNDING}

This work was supported by a National Research Foundation of Korea (NRF) grant to J-W Kim, funded by the Korean Government (2017R1D1A1B03031483).

\section{REFERENCES}

1. Perz JF, Armstrong GL, Farrington LA, Hutin YJ, Bell BP. The contributions of hepatitis $B$ virus and hepatitis $C$ virus infections to cirrhosis and primary liver cancer worldwide. J Hepatol. 2006; 45:529-538.

2. Bruix J, Sherman M, American Association for the Study of Liver D. Management of hepatocellular carcinoma: an update. Hepatology. 2011; 53:1020-1022.

3. European Association For The Study Of The Liver; European Organisation For Research And Treatment Of Cancer. EASL-EORTC clinical practice guidelines: management of hepatocellular carcinoma. J Hepatol. 2012; 56:908-943.

4. Omata M, Lesmana LA, Tateishi R, Chen PJ, Lin SM, Yoshida H, Kudo M, Lee JM, Choi BI, Poon RT, Shiina S, Cheng AL, Jia JD, et al. Asian Pacific Association for the Study of the Liver consensus recommendations on hepatocellular carcinoma. Hepatol Int. 2010; 4:439-474.

5. Bruix J, Sherman M. Management of hepatocellular carcinoma. Hepatology. 2005; 42:1208-1236.

6. Singal A, Volk ML, Waljee A, Salgia R, Higgins P, Rogers MA, Marrero JA. Meta-analysis: surveillance with ultrasound for early-stage hepatocellular carcinoma in patients with cirrhosis. Aliment Pharmacol Ther. 2009; 30:37-47.

7. Singal AG, Conjeevaram HS, Volk ML, Fu S, Fontana RJ, Askari F, Su GL, Lok AS, Marrero JA. Effectiveness of hepatocellular carcinoma surveillance in patients with cirrhosis. Cancer Epidemiol Biomarkers Prev. 2012; 21:793-799.

8. Sinn DH, Yi J, Choi MS, Kim YJ, Gwak GY, Lee JH, Koh KC, Paik SW, Yoo BC. Serum alpha-fetoprotein may have a significant role in the surveillance of hepatocellular carcinoma in hepatitis B endemic areas. Hepatogastroenterology. 2015; 62:327-332.

9. Yu NC, Chaudhari V, Raman SS, Lassman C, Tong MJ, Busuttil RW, Lu DS. CT and MRI improve detection of hepatocellular carcinoma, compared with ultrasound alone, in patients with cirrhosis. Clin Gastroenterol Hepatol. 2011; 9:161-167.

10. Bruix J, Reig M, Sherman M. Evidence-Based Diagnosis, Staging, and Treatment of Patients With Hepatocellular Carcinoma. Gastroenterology. 2016; 150:835-853.

11. Bolondi L, Cillo U, Colombo M, Craxì A, Farinati F, Giannini EG, Golfieri R, Levrero M, Pinna AD, Piscaglia F, Raimondo G, Trevisani F, Bruno R, et al; Italian Association for the Study of the Liver (AISF); AISF Expert Panel; AISF Coordinating Committee. Position paper of the Italian Association for the Study of the Liver (AISF): the multidisciplinary clinical approach to hepatocellular carcinoma. Dig Liver Dis. 2013; 45:712-723.

12. Deeks JJ, Altman DG. Diagnostic tests 4: likelihood ratios. Bmj. 2004; 329:168-169.

13. Jaeschke R, Guyatt GH, Sackett DL. Users' guides to the medical literature. III. How to use an article about a diagnostic test. B. What are the results and will they help me in caring for my patients? The Evidence-Based Medicine Working Group. JAMA. 1994; 271:703-707.

14. Yuen MF, Tanaka Y, Fong DY, Fung J, Wong DK, Yuen JC, But DY, Chan AO, Wong BC, Mizokami M, Lai CL. Independent risk factors and predictive score for the development of hepatocellular carcinoma in chronic hepatitis B. J Hepatol. 2009; 50:80-88.

15. Wong VW, Chan SL, Mo F, Chan TC, Loong HH, Wong GL, Lui YY, Chan AT, Sung JJ, Yeo W, Chan HL, Mok TS. Clinical scoring system to predict hepatocellular carcinoma in chronic hepatitis B carriers. J Clin Oncol. 2010; 28:1660-1665.

16. Yang HI, Yuen MF, Chan HL, Han KH, Chen PJ, Kim DY, Ahn SH, Chen CJ, Wong VW, Seto WK; REACH-B Working Group. Risk estimation for hepatocellular carcinoma in chronic hepatitis B (REACH-B): development and validation of a predictive score. Lancet Oncol. 2011; 12:568-574.

17. Papatheodoridis G, Dalekos G, Sypsa V, Yurdaydin C, Buti M, Goulis J, Calleja JL, Chi H, Manolakopoulos S, Mangia G, Gatselis N, Keskin O, Savvidou S, et al. PAGE-B predicts the risk of developing hepatocellular carcinoma in Caucasians with chronic hepatitis B on 5-year antiviral therapy. J Hepatol. 2016; 64:800-806.

18. Poh Z, Shen L, Yang HI, Seto WK, Wong VW, Lin CY, Goh BB, Chang PE, Chan HL, Yuen MF, Chen CJ, Tan CK. 
Real-world risk score for hepatocellular carcinoma (RWSHCC): a clinically practical risk predictor for $\mathrm{HCC}$ in chronic hepatitis B. Gut. 2016; 65:887-888.

19. Biselli M, Conti F, Gramenzi A, Frigerio M, Cucchetti A, Fatti G, D'Angelo M, Dall'Agata M, Giannini EG, Farinati F, Ciccarese F, Andreone P, Bernardi M, Trevisani F. A new approach to the use of alpha-fetoprotein as surveillance test for hepatocellular carcinoma in patients with cirrhosis. Br J Cancer. 2015; 112:69-76.

20. El-Serag HB, Kanwal F, Davila JA, Kramer J, Richardson P. A new laboratory-based algorithm to predict development of hepatocellular carcinoma in patients with hepatitis $\mathrm{C}$ and cirrhosis. Gastroenterology. 2014; 146:1249-1255 e1241.

21. Pencina MJ, D'Agostino RB Sr, Steyerberg EW. Extensions of net reclassification improvement calculations to measure usefulness of new biomarkers. Stat Med. 2011; 30:11-21.

22. Pencina MJ, D'Agostino RB Sr, D'Agostino RB Jr, Vasan RS. Evaluating the added predictive ability of a new marker: from area under the ROC curve to reclassification and beyond. Stat Med. 2008; 27:157-172; discussion 207-112.

23. Colli A, Fraquelli M, Casazza G, Massironi S, Colucci A, Conte D, Duca P. Accuracy of ultrasonography, spiral CT, magnetic resonance, and alpha-fetoprotein in diagnosing hepatocellular carcinoma: a systematic review. Am J Gastroenterol. 2006; 101:513-523.

24. Bolondi L, Sofia S, Siringo S, Gaiani S, Casali A, Zironi G, Piscaglia F, Gramantieri L, Zanetti M, Sherman M. Surveillance programme of cirrhotic patients for early diagnosis and treatment of hepatocellular carcinoma: a cost effectiveness analysis. Gut. 2001; 48:251-259.

25. Yu SJ. A concise review of updated guidelines regarding the management of hepatocellular carcinoma around the world: 2010-2016. Clin Mol Hepatol. 2016; 22:7-17.

26. Sohn W, Cho JY, Kim JH, Lee JI, Kim HJ, Woo MA, Jung SH, Paik YH. Risk score model for the development of hepatocellular carcinoma in treatment-naive patients receiving oral antiviral treatment for chronic hepatitis B. Clin Mol Hepatol. 2017; 23:170-178.

27. Kokudo N, Hasegawa K, Akahane M, Igaki H, Izumi N, Ichida T, Uemoto S, Kaneko S, Kawasaki S, Ku Y, Kudo M, Kubo S, Takayama T, et al. Evidence-based Clinical Practice Guidelines for Hepatocellular Carcinoma: The Japan Society of Hepatology 2013 update (3rd JSH-HCC Guidelines). Hepatol Res. 2015; 45.

28. Volk ML, Hernandez JC, Su GL, Lok AS, Marrero JA. Risk factors for hepatocellular carcinoma may impair the performance of biomarkers: a comparison of AFP, DCP, and AFP-L3. Cancer Biomark. 2007; 3:79-87.

29. Sherman M. Serological surveillance for hepatocellular carcinoma: time to quit. J Hepatol. 2010; 52:614-615.

30. Lok AS, Lai CL. alpha-Fetoprotein monitoring in Chinese patients with chronic hepatitis B virus infection: role in the early detection of hepatocellular carcinoma. Hepatology. 1989; 9:110-115.

31. Di Bisceglie AM, Hoofnagle JH. Elevations in serum alpha-fetoprotein levels in patients with chronic hepatitis B. Cancer. 1989; 64:2117-2120.

32. Lin YJ, Lee MH, Yang HI, Jen CL, You SL, Wang LY, Lu SN, Liu J, Chen CJ. Predictability of liver-related seromarkers for the risk of hepatocellular carcinoma in chronic hepatitis B patients. PLoS One. 2013; 8:e61448.

33. Wong GL, Chan HL, Tse YK, Chan HY, Tse CH, Lo AO, Wong VW. On-treatment alpha-fetoprotein is a specific tumor marker for hepatocellular carcinoma in patients with chronic hepatitis B receiving entecavir. Hepatology. 2014; 59:986-995.

34. Chang TS, Wu YC, Tung SY, Wei KL, Hsieh YY, Huang HC, Chen WM, Shen CH, Lu CH, Wu CS, Tsai YH, Huang YH. Alpha-Fetoprotein Measurement Benefits Hepatocellular Carcinoma Surveillance in Patients with Cirrhosis. Am J Gastroenterol. 2015; 110:836-844; quiz 845.

35. Andersson KL, Salomon JA, Goldie SJ, Chung RT. Cost effectiveness of alternative surveillance strategies for hepatocellular carcinoma in patients with cirrhosis. Clin Gastroenterol Hepatol. 2008; 6:1418-1424.

36. Giannini EG, Erroi V, Trevisani F. Effectiveness of alphafetoprotein for hepatocellular carcinoma surveillance: the return of the living-dead? Expert Rev Gastroenterol Hepatol. 2012; 6:441-444.

37. Vickers AJ, Cronin AM, Elkin EB, Gonen M. Extensions to decision curve analysis, a novel method for evaluating diagnostic tests, prediction models and molecular markers. BMC Med Inform Decis Mak. 2008; 8:53.

38. Vickers AJ, Elkin EB. Decision curve analysis: a novel method for evaluating prediction models. Med Decis Making. 2006; 26:565-574.

39. Choi J, Kim JW, Seo JW, Chung CK, Kim KH, Kim JH, Kim JH, Chie EK, Cho HJ, Goo JM, Lee HJ, Wee WR, Nam SM, et al. Implementation of Consolidated HIS: Improving Quality and Efficiency of Healthcare. Healthc Inform Res. 2010; 16:299-304.

40. Yoo S, Kim S, Lee KH, Baek RM, Hwang H. A study of user requests regarding the fully electronic health record system at Seoul National University Bundang Hospital. Stud Health Technol Inform. 2013; 192:1015.

41. Chung JW, Kim BH, Lee CS, Kim GH, Sohn HR, Min BY, Song JC, Park HK, Jang ES, Yoon H, Kim J, Shin CM, Park YS, et al. Optimizing Surveillance Performance of Alpha-Fetoprotein by Selection of Proper Target Population in Chronic Hepatitis B. PLoS One. 2016; 11:e0168189.

42. Yoo S, Lee KH, Lee HJ, Ha K, Lim C, Chin HJ, Yun J, Cho EY, Chung E, Baek RM, Chung CY, Wee WR, Lee $\mathrm{CH}$, et al. Seoul National University Bundang Hospital's Electronic System for Total Care. Healthc Inform Res. 2012; 18:145-152. 
43. Chan HL, Hui AY, Wong ML, Tse AM, Hung LC, Wong VW, Sung JJ. Genotype C hepatitis B virus infection is associated with an increased risk of hepatocellular carcinoma. Gut. 2004; 53:1494-1498.

44. Collins GS, Reitsma JB, Altman DG, Moons KG. Transparent Reporting of a multivariable prediction model for Individual Prognosis or Diagnosis (TRIPOD): the TRIPOD statement. Ann Intern Med. 2015; 162:55-63.

45. Moons KG, Altman DG, Reitsma JB, Ioannidis JP, Macaskill P, Steyerberg EW, Vickers AJ, Ransohoff DF, Collins GS. Transparent Reporting of a multivariable prediction model for Individual Prognosis or Diagnosis (TRIPOD): explanation and elaboration. Ann Intern Med. 2015; 162:W1-73.

46. Lemeshow S, Hosmer DW Jr. A review of goodness of fit statistics for use in the development of logistic regression models. Am J Epidemiol. 1982; 115:92-106.

47. Steyerberg EW, Vickers AJ, Cook NR, Gerds T, Gonen M, Obuchowski N, Pencina MJ, Kattan MW. Assessing the performance of prediction models: a framework for traditional and novel measures. Epidemiology. 2010; $21: 128-138$ 